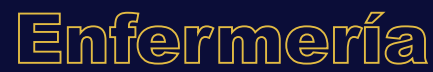

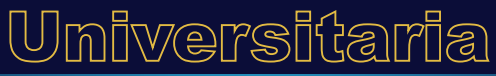

\section{Laboratorio MakerNurse, evaluando la experiencia de innovar en estudiantes de enfermería}

\section{MakerNurse lab, assessing the innovating experiences among nursing students}

\section{Laboratório MakerNurse, avaliando a experiência de inovar em estudantes de enfermagem}

\section{Campillay-Campillay ${ }^{\mathrm{a} 1}$, P. Dubó-Araya ${ }^{\mathrm{b}^{*} \text {, }}$ E. Rivas-Rivero ${ }^{c 2}$, A. Calle-Carrasco ${ }^{d_{3}}$, D. Aguilera-Ángel ${ }^{\mathrm{e}}$, F. Ponce-Pimentel ${ }^{\mathrm{f} 1}$}

ORCID

a $0000-0002-4054-1595$

${ }^{\mathrm{d}} \underline{0000-0002-8099-1486}$

${ }^{\mathrm{b}} 0000-0001-6918-3659$

e $0000-0002-2415-4026$

c0000-0002-9832-4534

${ }^{\mathrm{f}} 0000-0003-4134-0725$

${ }^{1}$ Universidad de Atacama, Facultad Ciencias de la Salud, Departamento de Enfermería, Atacama, Chile

${ }^{2}$ Universidad de La Frontera, Facultad de Medicina, Departamento de Enfermería, Araucanía, Chile

${ }^{3}$ Universidad de Atacama, Facultad Ciencias de la Salud, Departamento de Kinesiología, Atacama, Chile

Recibido: 23 mayo 2020

Aceptado: 09 septiembre 2020

\section{RESUMEN}

Introducción: Los MakerNurse son laboratorios que recogen la tradición del inventor giro sin tornillo y tecnologías de la información de alcance global. En este espacio disruptivo se ha

*Autor para correspondencia. Correo electrónico: pablo.dubo@uda.cl https://doi.org/10.22201/eneo.23958421e.2021.1.900

1665-7063/@ 2021 Universidad Nacional Autónoma de México, Escuela Nacional de Enfermería y Obstetricia. Este es un artículo Open Access bajo la licencia CC BY-NC-ND (http://creativecommons.org/licenses/by-nc-nd/4.o/). 
implementado un taller lectivo para la enseñanza de la innovación y el emprendimiento en estudiantes de enfermería y otras carreras de la salud.

Objetivo: Evaluar la experiencia de instalar un MakerNurse en la carrera de enfermería de la Universidad de Atacama.

Metodología: Se utiliza el estudio de caso con abordaje cualitativo-cuantitativo (CUAL-cuan) con mayor énfasis en lo cualitativo. La fase cuantitativa es descriptiva transversal, y la fase cualitativa es interpretativa con categorías a priori para resaltar aspectos de la instalación del laboratorio. Se utilizaron los datos correspondientes a los cursos realizados y a los proyectos adjudicados.

Discusión: Los principales usuarios de los laboratorios a nivel global son jóvenes de menos de 20 años, nativos tecnológicos, con capacidad para generar redes de comunicación global. El MakerNurse proporciona un espacio de enseñanza-aprendizaje en diseño y fabricación de prototipos, rediseño de procesos clínicos y formulación de proyectos para lograr mejores cuidados.

Resultados: El laboratorio MakerNurse dicta talleres sobre innovación y emprendimiento de manera regular, y como resultado se han generado 48 proyectos de variadas temáticas, 6 de estos lograron adjudicación de fondos del Ministerio de educación.

Conclusión: El laboratorio MakerNurse ha sido una buena estrategia para desarrollar competencias en innovación y emprendimiento estudiantil en enfermería.

Palabras clave: Enfermería; creatividad; rol de la enfermera; laboratorios; Chile.

\section{ABSTRACT}

Introduction: MakerNurse are labs which follow the tradition of turn without bolt and information technologies with worldwide reach. In our space, a workshop has been established to foster teaching innovation and entrepreneurship to students of health sciences, including nursing.

Objective: To assess the experience of implementing a MakerNurse lab in the nursing program of the University of Atacama, Chile.

Methodology: Case study with a qualitative-quantitative approach. The quantitative methodology is descriptive and transversal. The qualitative methodology is pre and post lab implementation interpretation. Data on given courses and accepted projects were used. Discussion: The main users of this kind of labs are students around 20 years old and who are knowledgeable in technology and communication networks. The MakerNurse lab offers a teaching-learning space to design prototypes, refine clinical processes, and create projects, all aimed at improving healthcare.

Results: From the implementation of the MakerNurse lab, 48 projects with diverse objectives have been generated. Six of these projects have received funding from the Ministry of Education.

Conclusion: The MarkerNurse lab has been a good strategy to develop competencies in innovation and entrepreneurship among nursing students.

Keywords: Nursing; criativity; nurse's role; laboratories; Chile. 


\section{RESUMO}

Introdução: Os MakerNurse são laboratórios que reúnem a tradição do inventor twist sem parafuso e tecnologias de informação de alcance global. Neste espaço disruptivo foi implementada uma oficina letiva para o ensino da inovação e o empreendedorismo em estudantes de enfermagem e outras carreiras de saúde.

Objetivo: Avaliar a experiência de instalação de um MakerNurse na carreira de enfermagem da Universidade de Atacama.

Metodologia: Foi utilizado o estudo de caso com abordagem qualitativo-quantitativa (CUALcuan) com maior ênfase na qualitativa. A fase quantitativa é descritiva transversal, e a fase qualitativa é interpretativa com categorias a priori para destacar aspectos da instalação do laboratório. Foram utilizados os dados correspondentes aos cursos realizados e aos projetos premiados.

Discussão: Os principais usuários dos laboratórios em nível global são jovens menores de 20 anos, nativos tecnológicos, com capacidade para gerar redes de comunicação global. A MakerNurse proporciona um espaço de ensino-aprendizagem em desenho e fabricação de protótipos, redesenho de processos clínicos e formulação de projetos para conseguir melhores cuidados.

Resultados: O laboratório MakerNurse ministra oficinas sobre inovação e empreendedorismo de forma regular, e como resultado foram gerados 48 projetos de diversas temáticas, 6 deles conseguiram concessão de fundos do Ministério de educação.

Conclusão: O laboratório MakerNurse tem sido uma boa estratégia para desenvolver competências em inovação e empreendedorismo discente em enfermagem.

Palavras chave: Enfermagem; criatividade; papel do profissional de enfermagem; laboratórios; Chile.

\section{INTRODUCCIÓN}

La innovación ha sido una fuente fundamental de progreso para las profesiones y una condición crítica para responder adecuadamente a cambios del entorno. La rapidez con que cambia el contexto sanitario, social y político, como resultado del proceso de globalización de las comunicaciones y el conocimiento, requiere de profesionales con capacidad para anticiparse a posibles riesgos para los pacientes y buscar maneras activas de mejorar la seguridad y calidad de los cuidados ${ }^{1-3}$.

La práctica profesional de enfermería se ha desarrollado clínicamente en un entorno altamente estandarizado y lineal, característica del modernismo que favorece la racionalidad utilitaria4. Esto ha inhibido el pensamiento innovador de estudiantes y profesionales, ha propiciado que expertos creativos mantengan sus ideas en un plano más bien informal, por lo que contar con una planificación para la acción innovadora o formalizar estrategias de enseñanza para innovar es un tema de relevancia ${ }^{1,5}$.

Transformar entornos rutinarios en creativos no es tarea sencilla y más difícil aún, conformar una cultura de innovación y emprendimiento para la profesión ${ }^{6}$. Para Van Holm7, un MakerSpace es un espacio democrático que facilita el acceso a la tecnología con el propósito de crear. Este movimiento social en torno a tecnologías de la información (TIC) favorece el pensamiento disruptivo e innovador en los estudiantes además del trabajo colaborativo por medio del uso de redes sociales digitales, para formar verdaderas comunidades virtuales del conocimiento ${ }^{8,9}$. Países desarrollados 
han utilizado estos laboratorios para la enseñanza y aprendizaje de ciencia e innovación permitiendo que la sociedad se beneficie de sus hallazgos. En este sentido, las universidades han tenido un efecto multiplicador de este modelo al promover redes de inventores de libre pensamiento y de las más variadas edades ${ }^{10}$.

Se considera que emprender es esencial para propiciar crecimiento y dinamismo a las profesiones, estos espacios han generado un alto grado de interés facilitado por el acceso casi universal a internet y la creciente disponibilidad de herramientas tecnológicas a menor costo ${ }^{11,12}$. Estas tecnologías se han vuelto indispensables en el avance profesional al optimizar tiempos y permitir mayor eficiencia en los procesos clínicos. Por lo tanto, los Maker entrelazan capacidades humanas creativas y sensibles a herramientas tecnológicas que permiten compartir el gusto por diseñar procesos o productos con el fin de beneficiar a la sociedad7,13,14.

El movimiento Maker incorpora, además, valores como compartir el conocimiento, colaborar con otros y aprender a partir de la búsqueda de soluciones en equipos de trabajo inter o transdisciplinario ${ }^{14}$. Los primeros MakerSpace liderados por enfermeras han tomado el nombre de MakerNurse y nacieron en hospitales magnéticos en 2013 en los Estados Unidos de América. Durante su funcionamiento han favorecido la disminución de costos operativos al proporcionar herramientas y tecnología en espacios de libre acceso para participantes de distintas disciplinas. El flujo de conocimiento transdisciplinario rompe fronteras entre las disciplinas aumentando las posibilidades creativas para mejorar la práctica clínica actual. Además, para las enfermeras es una forma de trabajo incipiente que ha demostrado buenos resultados por la gran motivación que genera entre los participantes, quienes por sí mismos realizan gestiones, establecen sus horarios, buscan capacitarse en el uso de herramientas tecnológicas y apoyo en personas más experimentadas, lo que facilita que estudiantes y profesionales se atrevan a experimentar con sus propias ideas , $74,15^{\text {. }}$.

Formar líderes que promuevan ideas diferentes es una conducta disruptiva que genera tensiones entre los profesionales que buscan cambios y otros que optan por mantener lo que existe de manera más conservadora. En este sentido, desarrollar una cultura que fomente la innovación deberá considerar la construcción de nuevas formas de ejercer el cuidado y adaptarse a cambios sociales globales, y al mismo tiempo mantener los aspectos sustanciales del cuidado ${ }^{16}$. Aunque los profesionales de enfermería han favorecido siempre el entorno creativo ${ }^{5,17}$, se han adaptado con lentitud a las exigencias del mundo globalizado y tecnológico, lo que ha hecho poco visible los aportes productivos realizados a nivel profesional ${ }^{18-20}$.

Al respecto, Marshall ${ }^{20}$ menciona que el ingenio de los profesionales de enfermería ha sido documentado en distintos lugares y momentos históricos del mundo moderno. Esta naturaleza creativa se ha observado con mayor fuerza cuando se ha actuado en entornos desfavorables y carentes de recursos. Un ejemplo identificado en Latinoamérica es el caso de enfermeras nicaragüenses que fueron descritas como especialmente creativas frente a la crisis económica de su país, pues al no contar con insumos necesarios para brindar cuidados, utilizaron todo tipo de materiales disponibles, entre ellos cartones de cajas de cereales con los que desarrollaron fijaciones que contribuyeron a mejorar las condiciones de los pacientes hospitalizados. Como se observa, el campo de la enfermería puede formalizar el cultivo de ideas, desarrollo de procesos y fabricación de productos.

Con base en lo anterior, el siguiente trabajo pretende responder a la pregunta: ¿cómo ha sido la experiencia de instalar un MakerNurse para estudiantes de enfermería en la Universidad de Atacama? Como antecedente se menciona que en el año 2016 surge la idea de crear un laboratorio que diera respuesta a problemáticas y brechas que detectaban los estudiantes en sus prácticas 
clínicas. Asimismo, ha sido resultado de un trabajo iniciado por un equipo de enfermería que planteó la idea al Fondo de Desarrollo Institucional para estudiantes (FDI) del Ministerio de Educación de Chile (MINEDUC), concurso que permitió implementar el laboratorio, cuyos beneficiarios han sido cuatro cohortes de estudiantes de pregrado de la Facultad de ciencias de la salud.

La evaluación se realiza a través de un estudio de caso único con metodología mixta (CUALcuan). Cabe destacar que esta investigación se considera un aporte al conocimiento disciplinar por las posibilidades de promover la innovación y desarrollo (I+D) en estudiantes. Además, se plantea como una temática emergente y relevante en el mundo globalizado.

\section{METODOLOGÍA}

Se realiza una evaluación de tipo mixta por medio del estudio de caso único ${ }^{21}$ para responder al objetivo general de evaluar la experiencia de instalar un MakerNurse durante el periodo 2016-2019 en el departamento de enfermería en la Universidad de Atacama. De acuerdo con Hernández et al.22, el estudio de caso como metodología permite ampliar la visión del fenómeno ya que aborda procesos holísticamente (conteo de ocurrencia, descripción de su estructura y sentido de entendimiento). El enfoque mixto bajo esta perspectiva incluye un abordaje CUAL-cuan con mayor énfasis en lo cualitativo, y aplicación de ambos métodos de manera simultánea. La fase cuantitativa es descriptiva transversal, cuya unidad de estudio es el Laboratorio MakerNurse, mientras que la fase cualitativa permite un análisis del contexto y un abordaje más reflexivo de las dimensiones consideradas.

Dado que la unidad de estudio y caso único es el laboratorio MakerNurse, se trabaja con el universo, este corresponde a los proyectos adjudicados de donde se obtienen los datos para la evaluación que considera las siguientes dimensiones: a) clasificación de los proyectos adjudicados por el laboratorio, b) género de los/as directores estudiantiles, c) características personales de directores de proyectos, d) principales actividades del laboratorio y e) resultados obtenidos (Tabla 1).

Se plantea como supuesto que el MakerNurse es una estrategia eficiente utilizada en el desarrollo de competencias para la innovación en estudiantes de enfermería. El sesgo principal se relaciona con que los/as autores/as del manuscrito corresponden a los/as académicos/as y estudiantes que participaron de la creación del MakerNurse. El documento cumple con los aspectos éticos de la investigación al omitir datos sensibles y considerar el valor social de la experiencia.

\section{RESULTADOS}

En la tabla 1 se resume las dimensiones consideradas para la evaluación durante el periodo de cuatro años de experiencia comprendido entre 2016 y 2019: a) clasificación de los proyectos adjudicados por el laboratorio, b) género de los/as directores estudiantiles, c) características personales de directores de proyectos, d) principales actividades del laboratorio y e) resultados obtenidos.

Durante el periodo evaluado, el laboratorio promovió ocho cursos en modalidad presencial para estudiantes de enfermería y ciencias de la salud, se alcanzó una cobertura de 240 estudiantes en el periodo revisado, de estos $85 \%$ fueron mujeres y $15 \%$ hombres. Como resultado de trabajos de finalización de curso se recibieron 48 proyectos relacionados con temas sanitarios con enfoque social. Diez de ellos fueron presentados a fondos nacionales del MINEDUC, donde seis lograron obtener financiamiento para su ejecución. Los proyectos contaron con el auspicio de dos académicas quienes participaron del diseño, seguimiento y cierre administrativo de cada proyecto. Estos se agruparon en tres áreas de trabajo diferentes; a) ciencia y tecnología e innovación, b) trabajo social con vinculación comunitaria, y c) inclusión de personas con discapacidad. 
El género de los directores de proyecto muestra una mayor tendencia hacia lo masculino, ya que sólo dos de seis proyectos fueron dirigidos por mujeres. Los directores estudiantiles pertenecían a distintas cohortes de estudiantes de enfermería y otras carreras de la salud. Tres de los directores estudiantiles tenían experiencia previa con proyectos, quienes además lideraron la formación de monitores en sus áreas de conocimiento; uso de tecnologías $3 \mathrm{D}$, risoterapia y lengua de señas chilena.

Las principales actividades planificadas en las propuestas tuvieron una secuencia lógica que inició con el primer proyecto que tuvo como objetivo la instalación del laboratorio, la elección y adquisición de herramientas y equipos, procesos de comunicación formal, conectividad y reglas de funcionamiento del espacio. Una segunda etapa, de formación y capacitación de monitores, se consideró relevante para generar capital humano con competencias formales en innovación y emprendimiento, mientras que otras temáticas fueron establecidas de acuerdo con motivaciones e intereses de los estudiantes. Otra actividad clave fue la creación de cursos electivos para enfermería y otras carreras de la salud, con esto se aseguró un número de inscritos permanente y una asistencia de 90\% en promedio. Se realizaron cuatro electivos diferentes: emprendimiento e innovación, lengua de señas básico, risoterapia y lecto escritura Braille (sólo el curso de emprendimiento e innovación se dictó de manera regular). Este aspecto se considera relevante, pues ha generado una masa crítica de estudiantes interesados en innovar, quienes mantienen una vinculación permanente con el laboratorio.

La implementación de la infraestructura del laboratorio incluyó: salón amplio, mesas redondas con capacidad para 20 personas, impresora 3D, escáner de imagen, plotter, herramientas de corte y pulido, impresora Braille, cámaras fotográficas y de vídeo, internet con acceso a bases de datos de revistas de alto impacto, computadores, programas de diseño y análisis de datos, disfraces de payasos, pizarras en varios lugares, máquina dispensadora de agua y café, entre muchos otros materiales para actividades manuales.

Se establecieron procesos de funcionamiento del laboratorio, cuya única regla ha sido cuidar los materiales y herramientas, ya que su uso no está restringido a horarios sino al interés y motivación de los participantes. Otro aspecto de los proyectos es que tres de éstos evolucionaron hacia tesis de grado de licenciaturas; dos en enfermería y una de kinesiología en conjunto con enfermería. La vinculación con el medio es otro aspecto que ha sido fundamental en el avance del laboratorio, pues la interacción con el entorno clínico donde los alumnos/as realizan prácticas ha generado visibilidad de las ideas priorizando problemáticas del medio, lo que agrega valor social y disciplinar al conectar el entorno académico con la práctica cotidiana sanitaria.

La metodología de trabajo ha sido el pilar central del espacio MakerNurse basado en el acompañamiento de un mentor/a encargado de motivar y guiar el trabajo de los/as estudiantes. En efecto, estos deben pensar en la idea o el problema a solucionar basado en su experiencia de práctica clínica, para luego hacer un diagnóstico de la situación actual, comparando con soluciones existentes en el mercado. Para ello, se utilizó el modelo Design thinking basado en la creación de prototipos y desarrollo de propuestas de soluciones originales con énfasis en las personas. Posteriormente, los grupos de estudiantes desarrollan el Canvas, que corresponde al modelo de negocios que mejor se ajusta al producto o proceso creado.

Los resultados obtenidos son presentados como pitch (propuesta) frente a una comisión de académicos/as invitados/as quienes evalúan el proyecto a partir de una rúbrica ad hoc ${ }^{14}$. Este proceso iterativo de enseñanza-aprendizaje es condición para la aprobación de la asignatura y promueve 
que posteriormente todos los grupos busquen fondos para ejecutar sus proyectos. Junto a la generación de conocimiento y habilidades en I+D, los estudiantes adquieren habilidades de comunicación, trabajo en equipo y sensibilidad ética al tener que fundamentar el bien social involucrado en la solución planteada.

\section{DISCUSIÓN}

\section{a) Clasificación de los proyectos}

La innovación y emprendimiento es la temática principal del laboratorio MakerNurse, ya que constituye la base que permite trabajar cualquier tema en el ámbito del desarrollo de productos o servicios sanitarios. Los proyectos adjudicados vinculan al laboratorio con comunidades vulnerables, especialmente personas con discapacidad, personas mayores, niños/as y mujeres. En este sentido, las temáticas de los proyectos responden al modelo de justicia social promocionado por organizaciones de derechos humanos a nivel internacional, abordando la búsqueda de soluciones a problemas que afectan a la población mundial por igual23,24.

Al respecto, la literatura coincide en que el entorno de la profesión de enfermería favorece la innovación, sin descuidar el valor social de sus acciones ${ }^{20}$. Los rasgos característicos de las enfermeras/ os facilitan su trabajo creativo, por ejemplo, Flinter et al. ${ }^{23}$ mencionan que capacidades como traducir las necesidades de los pacientes, así como las instrucciones médicas, han sido fundamentales para crear procesos y productos para el cuidado.

Por otra parte, las TIC son de uso obligatorio para acceder al laboratorio, ya que son el medio universal de comunicación, tal como lo mencionan Vicens et al. ${ }^{25}$ al experimentar exitosamente con laboratorios de comunidades globales en donde se obtuvo una alta participación y en diversos grupos de edades. Las TIC ciertamente contribuyen a la formación de individuos críticos, creativos y competentes para la vida y el trabajo, de modo que provocan nuevas exigencias a la academia y al entorno laboral ${ }^{26}$. El desarrollo de plataformas en internet con el fin de divulgar la ciencia, intercambiar ideas y realizar investigación transnacional, resalta aspectos de cooperación entre personas que no se conocen físicamente, pero definen el rasgo de los que participan en el uso de estas plataformas ${ }^{23}$. De esta forma, los Maker utilizan espacios educativos democráticos que brindan a los estudiantes la oportunidad de ampliar e impulsar sus capacidades creativas individuales y colectivas abordando áreas de gran interés social ${ }^{26}$.

\section{b) Género de los/as directores de proyecto}

Como se mencionó anteriormente, se ha podido observar mayor participación de directores estudiantes de género masculino que femenino, a pesar de que la profesión de enfermería es una carrera altamente feminizada. El conflicto entre género y liderazgo, según Mancayo y Zuluaga ${ }^{27}$, ha empezado a ser desafiado a partir de las últimas dos décadas, aunque se mantienen juicios peyorativos hacia mujeres que muestran cualidades de líderes, repitiéndose patrones donde a los hombres se les han asignado roles sociales de mayor liderazgo. En este aspecto, el laboratorio favorece la participación de mujeres como líderes y ejecutoras de proyectos de innovación y emprendimiento.

Además, dos de los tres guías de proyectos actuales son académicos mujeres lo que favorece el aprendizaje vicario y la influencia de modelos disruptivos. Es también materia de revisión que exista una auto discriminación de los propios estudiantes mujeres, quienes privilegian actividades académicas convencionales por sobre las no convencionales, respondiendo en esta forma a lo que espera la sociedad de ellas. En este sentido, el laboratorio facilita el trabajo en un entorno diverso, pluralista 
y reconoce liderazgos sin discriminación de ningún tipo, aspectos relevantes para el ejercicio del rol de los futuros gestores del cuidado.

\section{c) Características personales de los/as directores de proyectos}

En el entorno académico, la participación en estos laboratorios está restringida principalmente a los estudiantes que, dadas las características de las universidades, se consideran ideales para el desarrollo de la creatividad y cultivo del conocimiento. Los estudiantes inician su participación en promedio con menos de 20 años de edad, nativos tecnológicos que pertenecen a la generación postmilenio, personas que dominan las tecnologías de manera intuitiva y buscan obtener resultados de manera rápida. Son miembros de las redes sociales con manejo digital sobresaliente, ventaja que facilita su adaptación casi espontánea al entorno Maker.

Esto último coincide con la literatura revisada, en la que se plantea que los participantes de los Maker esencialmente cuentan con habilidades digitales y desarrollan cualidades en investigación y habilidades sociales al encontrar en estos espacios un lugar idóneo para emprender y experimentar; así como para ensayar con nuevas metodologías, fórmulas educativas y participación ciudadana ${ }^{13}$. En países desarrollados, los Maker han evolucionado hacia Social-Lab, variaciones de los Maker que desarrollan la cultura digital y tecnológica con fines específicamente sociales. Estos laboratorios pretenden, según Romero et al., [...] servir como nexo entre la sociedad y la academia, convirtiéndose en un espacio de cocreación y colaboración ciudadana ${ }^{13}$, lo que les da un carácter docente y de divulgación al conformar un canal bidireccional a través del cual ciudadanos e investigadores se influyen mutuamente y comparten conocimiento.

\section{d) Principales actividades del laboratorio}

El MakerNurse favorece el trabajo en red y proporciona el contexto adecuado para que estudiantes de enfermería puedan concretar ideas, diseñar procesos y productos, saliendo del sigilo de las unidades clínicas a una escala que pueda ser global. Además, proporciona capacitación en herramientas y tecnología para crear e intercambiar soluciones para la práctica clínica ${ }^{28}$. Junto con estas actividades, los estudiantes reciben demostraciones de cómo diseñar y fabricar prototipos, repensar procesos clínicos-administrativos que brindan la posibilidad de practicar y equivocarse, así como favorece el autoaprendizaje o aprendizaje entre pares. Esto fue descrito también por otros Maker que independientemente de su propósito o énfasis coinciden en los beneficios de este tipo de experiencias ${ }^{14}$.

Según Stein ${ }^{6}$, la relevancia de lo planteado convoca a la academia a cumplir el papel fundamental de realizar el proceso formativo de emprendedores en la profesión de enfermería. Este aspecto contribuye a promover iniciativas en los estudiantes, amplía las posibilidades de intervenir en el mercado laboral y contribuye a evaluar permanentemente la posibilidad de ampliar el rol profesional. Desde esta perspectiva, Ronquillo et al. ${ }^{29}$ agregan que incursionar en la informática y tecnologías de la información para la profesión ha producido incertidumbre, ya que el colectivo aún no aclara o delimita la forma en que esto contribuye a satisfacer las necesidades de los profesionales contemporáneos. Las tecnologías desde estos puntos de vista constituyen un desafío para el colectivo al tener que ponerse en movimiento para ampliar el uso de las TIC y así favorecer el conocimiento disciplinar.

\section{e) Resultados obtenidos}

Se relacionan con el desarrollo de habilidades avanzadas en el uso de tecnologías de la información, como se ha mencionado, éstas incluyen herramientas antes utilizadas sólo por ingenieros o 
profesionales afines, como la impresora láser 3D, escáner, programas de diseño gráfico y computadores Mac, entre los principales. Estos resultados educativos demuestran que es posible que enfermeras/os y otras profesiones sanitarias adquieran competencias para el diseño y fabricación de prototipos ${ }^{14}$. Los estudiantes aplican estos conocimientos en su propio proyecto, el que presentan como prototipo al final del curso.

De acuerdo con Lorenzini ${ }^{30}$, el uso de estas herramientas tecnológicas y talleres de innovación es poco frecuente en el área sanitaria, pero fundamental como aporte para transformar ideas y conocimiento en nuevos productos, procesos, modelos y sistemas para el beneficio de las personas y el desarrollo de la investigación aplicada en enfermería. En este sentido, el espacio físico, infraestructura y conectividad debe cumplir con los estándares que tienen los MakerSpace ${ }^{13}$.

Trabajar conformando equipos inter o transdisciplinarios ha sido otra condición de la nueva forma de construir conocimiento en el MakerNurse, esto ha favorecido el intercambio de ideas, ha estimulado el pensamiento crítico ampliado y ha mejorado en los estudiantes la comunicación y el liderazgo. Geist et al..$^{15}$ realizaron una experiencia en la cual alumnos de enfermería e ingeniería en un ejercicio clínico de laboratorio Maker lograron desarrollar prototipos funcionales innovadores y con posibilidades comerciales. En el MakerNurse se han auspiciado ocho proyectos y de estos seis han obtenido fondos a nivel nacional para su ejecución, se ha presentado una patente que está en evaluación, y se han creado programas de cuidados enfermeros que se escapan al cuidado convencional de la profesión.

Autores influyentes en el pensamiento de enfermería como Callista Roy ${ }^{16}$, han señalado que, con el rápido desarrollo de otras ciencias, la academia de enfermería tiene el desafío de buscar cómo crear nuevo conocimiento científico para la práctica; considerando nuevos contextos, pero sin perder de vista el desarrollo teórico y empírico que ha posicionado a enfermería a nivel profesional en el mundo. Asimismo, la enfermería debe contribuir a mejorar la adopción de mejores prácticas en la comunidad, mejorar el cuidado y la atención en salud en primer lugar, ya que cualquier conocimiento emergente podría ser también una tentación y desviar a la profesión de su sentido. El enfoque unificador propuesto por Roy permite no perder de vista los objetivos que nos unen como profesión, cuyo sustento teórico es tan sólido y necesario que debe estar presente en cualquier nueva propuesta de roles sociales.

Con base en lo anterior, utilizar el concepto de MakerNurse es quizás una vía para mantener identidad, pero sin dejar de reconocer la importancia del trabajo colectivo y transdisciplinario en la práctica clínica. El esfuerzo debe considerar cambiar y mantenerse a la vez en el camino de la mejora continua del cuidado, con el propósito de que estos profesionales no se queden en segundo plano en la era del mundo globalizado ${ }^{31}$.

\section{CONCLUSIÓN}

La experiencia de instalar un MakerNurse es una tarea compleja porque rompe el esquema de los laboratorios tradicionales de enfermería. Sin embargo, permite que estudiantes de enfermería puedan capacitarse y recibir el acompañamiento necesario para crear, emprender y sacar adelante proyectos I+D con potencial social o comercial. Desde la perspectiva de los/as autores, las universidades son los lugares propicios para su enseñanza formal. Por consiguiente, se propone implementar MakerNurse en escuelas de enfermería, como estrategia educativa para estimular la experimentación e innovación, de forma que sea posible el desarrollo de productos y procesos para el cuidado, además de contribuir a transformar la profesión y aportar con nuevos roles sociales que respondan a la nueva era del conocimiento global. 
Tabla 1. Caracterización de proyectos adjudicados en MINEDUC ${ }^{32}$

\begin{tabular}{|c|c|c|c|c|c|}
\hline $\begin{array}{l}\text { Nombre del } \\
\text { proyecto }\end{array}$ & $\begin{array}{l}\text { Clasificación } \\
\text { del proyecto } \\
\text { (MINEDUC) }\end{array}$ & $\begin{array}{l}\text { Género del } \\
\text { estudiante }\end{array}$ & $\begin{array}{l}\text { Características } \\
\text { personales del } \\
\text { director/a } \\
\text { estudiantil }\end{array}$ & $\begin{array}{l}\text { Principales } \\
\text { actividades del } \\
\text { proyecto } \\
\text { (Resumido) }\end{array}$ & $\begin{array}{l}\text { Resultados } \\
\text { obtenidos }\end{array}$ \\
\hline
\end{tabular}

ATA $1505 \quad$ Ciencia, Masculino.

LIA: Laboratorio tecnología e en investigación innovación. aplicada para Investigación. estudiantes de la Facultad de ciencias de la salud. de enfermería. Primer proyecto. Manejo avanzado de tecnologías de la información. Sensibilidad social. ementar

laboratorio científico dirigido a los alumnos de la Facultad de ciencias de la salud, en el área de innovación tecnológica e investigación aplicada.
Implementación del laboratorio: Traspaso de la sala, compra de herramientas, equipos de computación, impresora 3D, scanner y otros. Se da forma de sala-taller interactivo. Realización de cursos de innovación, emprendimiento y uso de impresión 3D. Aumento de vinculación con el medio. Desarrollo de trabajo interdisciplinario.

\begin{tabular}{|c|c|c|c|}
\hline $\begin{array}{l}\text { ATA } 1605 \\
\text { Risoterapia, } \\
\text { hacia un trato } \\
\text { más humanizado } \\
\text { en los centros } \\
\text { de salud de } \\
\text { Copiapó. }\end{array}$ & $\begin{array}{l}\text { Trabajo social } \\
\text { y vinculación } \\
\text { comunitaria. }\end{array}$ & Masculino. & $\begin{array}{l}\text { Alumno de tercer año de Formar monitores } \\
\text { enfermería. Terapeuta en risoterapia. } \\
\text { en risoterapia con } \\
\text { certificación formal. } \\
\text { Experiencia en otros } \\
\text { proyectos comunitarios. } \\
\text { Manejo avanzado de } \\
\text { técnicas de intervención } \\
\text { social. }\end{array}$ \\
\hline
\end{tabular}

Se crea grupo dedicado a intervención comunitaria.

Aumento de vinculación con el medio. Desarrollo de trabajo interdisciplinario. Continuidad del trabajo a través de tesis de grado.

\begin{tabular}{|c|c|c|c|c|}
\hline $\begin{array}{l}\text { ATA } 1606 \\
\text { CCIES: } \\
\text { Capacitación } \\
\text { en ciencia, } \\
\text { emprendimiento } \\
\text { y salud. }\end{array}$ & $\begin{array}{l}\text { Innovación } \\
\text { tecnológica } \\
\text { de procesos } \\
\text { y prácticas } \\
\text { saludables. }\end{array}$ & asculino. & $\begin{array}{l}\text { Alumno de tercer año } \\
\text { de enfermería. Segundo } \\
\text { proyecto ganado. } \\
\text { Capacitado en el curso } \\
\text { de emprendimiento y } \\
\text { uso de impresora 3D. }\end{array}$ & $\begin{array}{l}\text { Diseñar electivo } \\
\text { para enseñanza } \\
\text { y aprendizaje de } \\
\text { competencias para } \\
\text { el emprendimiento } \\
\text { científico. }\end{array}$ \\
\hline
\end{tabular}

Se aprueba el electivo sobre emprendimiento e innovación en enfermería.

Se realiza curso con primer cohorte de alumnos que toman electivo.

Continuidad del trabajo a través de tesis de grado.

\begin{tabular}{|c|c|c|c|c|c|}
\hline $\begin{array}{l}\text { ATA } 1607 \\
\text { Mundo de señas } \\
\text { y su inclusión. }\end{array}$ & $\begin{array}{l}\text { Inclusión de } \\
\text { personas con } \\
\text { discapacidad. }\end{array}$ & Femenino. & $\begin{array}{l}\text { Alumna de cuarto año } \\
\text { de enfermería. Primer } \\
\text { proyecto. Experiencia } \\
\text { en trabajo con personas } \\
\text { sordas. Certificada } \\
\text { en lenguaje de señas } \\
\text { chileno básico. }\end{array}$ & $\begin{array}{l}\text { Realizar inventario } \\
\text { de señas en salud. } \\
\text { Realizar cursos de } \\
\text { lenguaje de señas } \\
\text { chileno básico. }\end{array}$ & $\begin{array}{l}\text { Se realiza inventario con } \\
\text { señas para el ámbito de } \\
\text { salud, en conjunto con } \\
\text { Asociación de sordos } \\
\text { Atacama. } \\
\text { Se realiza electivo de } \\
\text { lenguaje de señas } \\
\text { chileno para estudiantes } \\
\text { de la Facultad de salud. } \\
\text { Continuidad del trabajo } \\
\text { a través de tesis de } \\
\text { grado. }\end{array}$ \\
\hline
\end{tabular}


ATA 1706

Lecto escritura

Braille, visión

inclusiva en

salud.
Trabajo social y Femenino. Alumna de cuarto año vinculación con el medio. Inclusión de personas con discapacidad.

ATA 1805 Trabajo social y Masculino.

Conociéndonos, vinculación con

talleres para el el medio.

desarrollo social Inclusión de

de niños/as y personas con

adolescentes discapacidad.

con

discapacidad

mental.
Implementación de Compra de impresora

taller de Braille. Braille, regletas de escritura manual y papel.

Se realiza taller de escritura Braille.

Continuidad del trabajo

a través de tesis de grado.

Detección de barreras para inclusión escolar en colegios vulnerables. Difusión sobre derechos humanos de personas con Trabajo de tesis que cruza los objetivos del proyecto.

Se diseña material para la enseñanza de derechos humanos en los colegios que forman parte del estudio. discapacidad.

Fuente: Universidad de Atacama. Informes de proyectos institucionales. Atacama, Chile: Universidad de Atacama, FID-MINEDUC; 2020.

\section{RESPONSABILIDADES ÉTICAS}

Protección de personas y animales. Los autores declaran que para esta investigación no se han realizado experimentos en seres humanos y animales.

Confidencialidad. Los autores declaran que no se brindan datos que permitan identificar a los participantes.

Derecho a la privacidad y consentimiento informado. Los autores declaran adherirse a la legislación vigente en el país y normativas éticas internacionales de investigación, con respecto a la autonomía y derecho a la privacidad de las personas.

Conflicto de intereses. Los autores declaran no tener conflicto de intereses.

Financiamiento. Ninguno.

\section{REFERENCIAS}

1. Cianelli R, Clipper B, Freeman R, Goldstein J, Wyatt TH. The innovation road map: A guide for nurse leaders. Princeton, Nueva Jersey: Robert Wood Johnson Foundation; 2016.

https://bit.ly/3sf8F3H

2. Kaya N, Turan N, Özdemir-Aydin G. Innovation in nursing: A concept analysis. J Comm Pub Health Nursing. 2016; 2(1): 1-4. http://dx.doi.org/10.4172/2471-9846.1000108

3. Grace PJ, Willis DG, Roy C, Jones DA. Profession at the crossroads: A dialog concerning the preparation of nursing scholars and leaders. Nurs Outlook. 2016; 64(1): 61-70.

http://dx.doi.org/10.1016/j.outlook.2015.10.002

4. Beltrán-Villegas MÁ, Cardona-Acevedo M. La sociología frente a los espejos del tiempo: modernidad, postmodernidad y globalización. Rev. Univ. EAFIT. 2005; 28: 1-79. https://bit.ly/3f2JwFA

5. Fasolino T. Addressing challenges of patients with chronic pulmonary disease using makerspace environments. Creat Nurs. 2019; 25(1): 46-52. https://doi.org/10.1891/1078-4535.25.1.46

6. Stein-Backes D. Emprendimiento en enfermería: estrategia proponente de un nuevo pensar y actuar. Av. enferm. 2018; 36(2): 141-2. https://doi.org/10.15446/av.enferm.v36n2.75934 
7. Van-Holm EJ. Makerspaces and contributions to entrepreneurship. Procedia Soc Behav Sci. 2015; 195: 24-31. https://doi.org/10.1016/j.sbspro.2015.06.167

8. Thomas TW, Seifert PC, Joyner JC. Registered nurses leading innovative changes. Online J Issues Nurs. 2016; 21(3): 3. https://bit.ly/3gKYala

9. Garaizar P, Reips UD. Build your own social network laboratory with Social Lab: A tool for research in social media. Behav Res Methods. 2014; 46(2): 430-8.

https://doi.org/10.3758/s13428-013-0385-3

10. Carucci K, Toyama K. Making well-being: exploring the role of makerspaces in long-term care facilities. CHI. 2019; 469: 1-12. https://doi.org/10.1145/3290605.3300699

11. García-Sáez C. Cómo hacer un espacio maker. Madrid: Gobierno de España/educa LAB; 2016. https://bit.ly/2P4okno

12. Bisballe-Jensen M, Sole-Semb CC, Vindal S, Steinert M. State of the art of makerspaces-success criteria when designing makerspaces for Norwegian industrial companies. Procedia CIRP. 2016; 54: 65-70. https://doi.org/10.1016/j.procir.2016.05.069

13. Romero-Frías R, Robinson-García N. Laboratorios sociales en universidades: Innovación e impacto en Medialab UGR. Comunicar. 2017; 25(51): 29-38. https://doi.org/10.3916/C51-2017-03

14. Taheri P, Robbins P, Maalej S. Makerspaces in first-year engineering education. Educ. Sci. 2020; 10(1): 1-16. https://doi.org/10.3390/educsci10010008

15. Geist MJ, Sanders R, Harris K, Arce-Trigatti A, Hitchcock-Cass C. Clinical immersion: An approach for fostering cross-disciplinary communication and innovation in nursing and engineering students. Nurse Educ. 2019; 44(2): 69-73. https://doi.org/10.1097/NNE.0000000000000547

16. Roy C. Nursing knowledge in the 21st century: Domain-derived and basic science practice-shaped. ANS Adv Nurs Sci. 2019; 42(1): 28-42. https://doi.org/10.1097/ANS.0000000000000240

17. Brysiewicz P, Hughes TL, McCreary LL. Promoting innovation in global nursing practice. AJOL. 2015; 2(2): 41-5. http://dx.doi.org/10.4314/rj.v2i2.7F

18. Behn-Theune V, Jara -Concha P, Nájera RM. Innovaciones en la formación del licenciado en enfermería en latinoamerica, al inicio del siglo XXI. Inv. educ. enferm. 2002; 20(2):48-56. https://bit.ly/3tMyUyx

19. Arandojo-Morales MI. Nuevas Tecnologías y nuevos retos para el profesional de enfermería. Index Enferm. 2016; 25(1-2): 38-41. https://bit.ly/39pI8AA

20. Marshall DR, McGrew DA. Creativity and innovation in health care: Opening a hospital makerspace. Nurse Lead. 2017; 15(1): 56-8. https://doi.org/10.1016/j.mnl.2016.10.002

21. Urra-Medina E, Núñez-Carrasco R, Retamal-Valenzuela C, Jure-Cares L. Enfoques de estudio de casos en la investigación de enfermería. Cienc. enferm. 2014; 20(1): 131-42. http://dx.doi.org/10.4067/S0717-95532014000100012

22. Hernández-Sampieri R, Mendoza-Torres CP. Metodología de la investigación. Las rutas cuantitativa, cualitativa y mixta. México: Mc Graw Hill Education; 2018.

23. Flinter M, Hsu C, Cromp DM, Ladden MD, Wagner EH. Registered nurses in primary care. Emerging new roles and contributions to team-based care in high-performing practices. J Ambul Care Manage. 2017; 4O(4): 287-96. https://doi.org/10.1097/JAC.0000000000000193

24. Simpson SD, Jakubec SL, Zawaduk C, Lyall C. Integrating global citizenship through local teaching practices. QANE-AFI. 2015; 1(2): 1-17. https://doi.org/10.17483/2368-6669.1031 
25. Vicens J, Perelló J, Duch J. Citizen social lab: A digital platform for human behavior experimentation within a citizen science framework. PLoS ONE. 2018; 13(12): 1-20.

https://doi.org/10.1371/journal.pone.0207219

26. Donato-Gottems LB, Alvarez AM, Wanderley-Santos de Almeida LM. Educação em Enfermagem: Qualidade, inovação e responsabilidade. Rev. bras. enferm. 2014; 67(4): 499-500. http://dx.doi.org/10.1590/0034-7167.2014670401

27. Moncayo-Orjuela BC, Zuluaga D. Liderazgo y género: barreras de mujeres directivas en la academia. Pensam. gest. 2015; 39: 142-77. https://bit.ly/3wNS49t

28. Gomez-Marquez J, Young A. A history of nurse making and stealth innovation. SSRN. 2016; 1-11. http://dx.doi.org/10.2139/ssm.2778663

29. Ronquillo C, Currie LM, Rodney P. The evolution of data-information-knowledge-wisdom in nursing informatics. ANS Adv Nurs Sci. 2016; 39(1): E1-18.

https://dx.doi.org/10.1097/ANS.0000000000000107

30. Lorenzini-Erdmann A. A Inovação em enfermagem. Cienc. enferm. 2013; 19(3): 7-9. http://dx.doi.org/10.4067/SO717-95532013000300001

31. Salmond SW, Echevarria M. Healthcare transformation and changing roles for nursing. Orthop Nurs. 2017; 36(1): 12-25. http://dx.doi.org/10.1097/NOR.0000000000000308

32. Universidad de Atacama. Informes de proyectos institucionales. Atacama, Chile: Universidad de Atacama, FID-MINEDUC; 2020. 